\title{
Tumor suppressor miR-424-5p abrogates ferroptosis in ovarian cancer through targeting ACSL4
}

\author{
Lin-Lin MA*, Lin LIANG*, Dan ZHOU, Shao-Wei WANG* \\ Department of Gynecology and Obstetrics, Beijing Hospital, National Center of Gerontology; Institute of Geriatric Medicine, Chinese Academy \\ of Medical Sciences, Beijing, China \\ ${ }^{*}$ Correspondence: $w \_s w 999 @ 163 . c o m$ \\ ${ }^{*}$ Contributed equally to this work.
}

Received July 7, 2020 / Accepted September 8, 2020

\begin{abstract}
Ovarian cancer is the most lethal gynecological cancer. In spite of recent advances, clinical outcomes remain poor, urgently needing novel therapeutic approaches. Growing evidence indicates that microRNAs play crucial roles in ovarian carcinogenesis and progression and that ferroptosis serves as a novel tumor suppressor. However, the molecular mechanisms of miRNA-mediated ferroptosis regulation in ovarian cancer are still largely unknown. In the present study, we show that miR-424-5p negatively regulates ferroptosis by directly targeting ACSL4 in ovarian cancer cells. Upregulation of miR-424-5p suppressed ACSL4 by directly binding to its 3'-UTR, which subsequently reduced erastin- and RSL3-induced ferroptosis. Meanwhile, knockdown of miR-424-5p increased the sensitivity of ovarian cancer cells to erastin and RSL3. Furthermore, ACSL4 was upregulated in ovarian cancer tissues, and high ACSL4 expression predicted worse prognosis and sensitized ovarian cancer cells to erastin- and RSL3-induced ferroptosis. Importantly, decreases in lipid peroxides and ferroptotic cell death mediated by miR-424-5p could be abrogated by ACSL4 overexpression. Taken together, our findings demonstrate that miR-424-5p regulates ferroptosis by targeting ACSL4 in ovarian cancer cells and suggest a potential therapeutic approach for ovarian cancer.
\end{abstract}

Key words: ovarian cancer, ACSL4, miR-424-5p, ferroptosis

Ovarian cancer is the most lethal gynecological cancer and ranks the fifth leading cause of cancer-associated deaths among women worldwide. For the absence of specific early symptoms, the majority of ovarian cancer patients are diagnosed at an advanced end-stage, with metastatic lesions beyond ovaries and a low 5-year survival rate [1]. Current standard ovarian cancer treatment is a combination of cytoreductive surgery and platinum-based combination chemotherapy [2]. Although ovarian cancer patients initially are sensitive to these treatments, the majority of patients will relapse and gradually develop platinum resistance [3]. Therefore, discovering novel effective therapeutic approaches for ovarian cancer patients is an urgent problem needing to be addressed.

miRNAs are small (20-24 nucleotides), endogenous non-coding RNAs that negatively regulate gene expression at the post-transcriptional level by binding to the 3 '-untranslated region (3'-UTR) of mRNAs, leading to translational inhibition or destabilization of target mRNAs [4]. Emerging evidence indicates that alternations in miRNAs are identi- fied to be related to various human cancers and that they function either as oncogenes or tumor suppressors owing to their critical roles in diverse biologic functions, such as cell proliferation, apoptosis as well as differentiation [5]. To date, a large number of miRNAs have been implicated in the development and progression of ovarian cancer $[6,7]$. Previous studies have shown that miR-424-5p was epigenetically downregulated in ovarian cancer tissues and cell lines owing to hypermethylation in the promoter regions and functioned as tumor suppressor miRNA by targeting oncogenes, KIF23 and CCNE1, respectively, and that decreased expression of miR-424-5p was significantly associated with distant metastasis in a high stage of ovarian cancer [8-10]. Moreover, several recent studies have demonstrated that downregulation of certain tumor suppressor miRNAs can render cancer cells extremely vulnerable to ferroptotic death, an iron-dependent form of non-apoptotic cell death driven by lethal lipid peroxidation, which may provide a potential and novel therapeutic strategy for cancer treatment [11-13]. Therefore, it is of 
interest to explore the role of miR-424-5p in the regulation of ovarian cancer cell ferroptosis.

Accumulating evidence has indicated that lipid metabolism contributes to the progression and chemoresistance of ovarian cancer and that alteration of cellular lipid composition is intimately involved in controlling ovarian cancer cell sensitivity to the initiation and execution of ferroptosis $[14,15]$. ACSL4 (Acyl-CoA synthetase long-chain family member 4 ) is an essential enzyme for the activation of the most abundant long-chain fatty acids (12-20 carbons), which facilitates biosynthesis of unsaturated phospholipids, the main substrates for lipid peroxidation [16]. Recent studies have shown that ACSL4 plays an oncogenic role in multiple cancer types and acts as a biomarker and contributor to ferroptosis [17-21]. However, its expression level, regulatory mechanism, and contribution to ferroptosis in ovarian cancer remain unknown. In this study, we explored the role of miR-424-5p in the control of ferroptosis and provided the first evidence that miR-424-5p regulated erastin- and RSL3-induced ferroptosis by directly targeting ACSL4. These data provide a potential therapeutic approach for the treatment of ovarian cancer.

\section{Patients and methods}

Clinical samples, cell lines, and transfection. A total of 38 pairs of ovarian cancer tissues and matched adjacent normal tissues were collected from Zhongnan Tumor Hospital, Wuhan, China. Tissues were obtained with informed consent and the current study was approved by local institutional review boards on human subject research and in accordance with the Declaration of Helsinki. All the histological diagnoses for ovarian cancer and normal tissues were reviewed and recognized by two pathologists independently. Human ovarian cancer cell lines HO8910 and SKOV3 were purchased from the American Type Culture Collection (Manassas, VA, USA) and cultured according to the provider's instruction. Both cell lines were tested free for Mycoplasma contamination by using Mycoplasma PCR Detected Kit (Sansure Biotech INC., P.R. China) according to the manufacturer's protocol. Transfection was performed with the Lipofectamine 2000 (Invitrogen, Carlsbad, CA, USA) according to the manufacturer's instructions.

Plasmids, siRNA, and microRNA. pcDNA3.1-ACSL4, ACSL4-specific siRNA, and miR-424-5p mimics/inhibitor were obtained from GenePharma (Shanghai, China). For luciferase reporter, the mRNA 3'UTR of ACSL4 containing the putative binding sites for miR-424-5p was obtained by PCR from human genomic DNA using the following forward primer: 5'-gtcatctagaAAATGTGTGAGTGCATGTA-3' and the reverse primer: 5'-gtcatctagaCTTGAAAAGTCACTATGGATATGGAAAG-3', both of which contain XbaI restriction site. The PCR product was digested with $\mathrm{XbaI}$ and subcloned into pGL3 luciferase reporter plasmid (Promega, Madison, WI, USA). Mutations in the miR-424-5p binding site of
ACSL4 3'UTR were produced by site-directed mutagenesis (Thermo Fisher, Waltham, MA, USA). Constructs were validated by DNA sequencing.

RNA isolation and qRT-PCR. Total RNA and miRNA were extracted from tissues and cells using RNeasy Mini and miRNeasy Mini Kits (Qiagen, Valencia, CA, USA) according to the manufacturer's instructions. Quantification of the expression of miR-424-5p was determined using a TaqMan miRNA assay kit (Thermo Fisher Scientific, Inc.). The expression of U6 was used as an endogenous control. Realtime PCR was performed using FastStart Universal SYBR Green Master kit (Roche Diagnostics, Mannhelm, Germany) and analyzed with an Applied Biosystems7900 Real-Time PCR System. Primer sequences were as follows: ACSL4, 5' GCTATCTCCTCAGACACACCGA-3' and 5'-AGGTGCTCCAACTCTGCCAGTA-3'; and $\beta$-actin, 5'-CACCATTGGCAATGAGCGGTTC-3' and ' 5 '-AGGTCTTTGCGGATGTCCACGT-3'. The relative expression levels of miR-424-5p and mRNAs were calculated and quantified using the $2^{-\Delta \Delta C T}$ method after normalization for the expression of the control. All the qRT-PCRs were run in triplicate.

Cell viability assay. Cell viability of control and treated (erastin, cat no. C101790; RSL3, cat no. C100988, ChemeGen USA) cells was examined using Cell Counting Kit-8 (CCK-8) (Dojindo Laboratories, Kumamoto, Japan) following the manufacturer's instructions. Absorbance at $450 \mathrm{~nm}$ was measured by a microplate reader. The viability assays were performed independently and repeated in triplicate.

Iron assay. Intracellular or mitochondrial ferrous iron $\left(\mathrm{Fe}^{2+}\right)$ level was measured by the iron assay kit obtained from Biovision (Milpitas, California, USA) according to the manufacturer's instructions.

Immunohistochemistry (IHC). Tissues were fixed, paraffin-embedded, and cut into $4 \mu \mathrm{m}$ sections. The sections were deparaffinized, rehydrated, and subjected to antigen retrieval using sodium citrate buffer. After blocking with $10 \%$ normal goat serum for $1 \mathrm{~h}$, the sections were incubated with ACSL4 antibody (sc-365230, Santa Cruz, USA) overnight at $4^{\circ} \mathrm{C}$. The subsequent procedures were performed according to the manufacturer's protocol.

Lipid peroxidation assay. The concentration of malondialdehyde (MDA), an end product of lipid peroxidation, was assessed using a lipid peroxidation colorimetric assay kit purchased from Biovision (Milpitas, California, USA) according to the manufacturer's instructions.

Western blotting. Total protein was prepared using a protein extraction kit (KGP9100, Key Gene). Equal amounts of protein were separated by SDS-PAGE, transferred onto PVDF membranes (Bio-Rad, Hercules, CA, USA), and blocked for $0.5 \mathrm{~h}$ at room temperature. The membranes were incubated with specific primary antibodies followed by secondary antibodies. The signals were detected using the Chemiluminescence HRP Substrate (WBKL0100, Millipore) and an enhanced chemiluminescence detection system. Antibodies against ACSL4 (ab205199, dilution 1/1000) 
were purchased from Abcam (Cambridge, USA), GAPDH (G9545, dilution 1/1000, Sigma Aldrich, St. Louis, USA) was used as a loading control.

Dual-luciferase assay. For the dual luciferase assay, ovarian cancer cells in a 96-well plate were transfected with

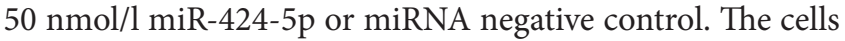
were then co-transfected with $0.2 \mathrm{mg} / \mathrm{ml}$ of plasmid with the wildtype or mutant 3'UTR of the ACSL4 gene. After $48 \mathrm{~h}$, luciferase activity was measured with the Dual-Luciferase reporter assay system (Promega). Firefly luciferase activity was then normalized to the corresponding Renilla luciferase activity. Luciferase assays were performed in quadruplicate and repeated in 3 independent experiments.

ACSL4 enzymatic activity assay. ACSL4 enzyme-specific activity was measured as previously described [22]. Briefly, the ACSL4 activity assay was performed with $50 \mu \mathrm{M}$ [1-14C] palmitic acid, $10 \mathrm{mM}$ ATP, and $0.25 \mathrm{mM} \mathrm{CoA}$ with total membrane factions (1-4 $\mu \mathrm{g}$ protein). Data were normalized per micrograms of protein determined by BCA assay.

5-HETE assay. 5-HETE, an ACSL4-mediated product of AA oxidation in cell lysates, was assessed using a 5-HETE ELISA kit (Uscn Life Science, Wuhan, China), according to the manufacturer's protocol.

Statistical analysis. SPSS17.0 statistical software package (SPSS, Chicago, IL, USA) was used for statistical analysis. Experiments were repeated independently at least three times, and the results are expressed as mean $\pm \mathrm{SD}$. The relationships between ACSL4 and clinicopathologic parameters were evaluated by a $\chi^{2}$ test. The correlation between miR-424-5p and ACSL4 was analyzed using Spearman's correlation test. Statistical differences between groups were evaluated using Student's paired two-tailed t-test. A p-value $<0.05$ was considered statistically significant.

\section{Results}

miR-424-5p inhibits erastin- and RSL3-induced ferroptosis in ovarian cancer cells. To explore the roles of miR-424-5p in the regulation of ovarian cancer ferroptosis, we performed gain- and loss-of-function experiments in ovarian cancer cell lines. The expression levels of miR-424-5p were significantly increased or decreased both in HO8910 and SKOV3 cells, when they were transfected with miR-424-5p mimics and miR-424-5p inhibitor, respectively (Supplementary Figure S1). Through cell viability assay, we found that erastin- and RSL3-induced cell death in both cell lines was significantly inhibited by the upregulation of miR-424-5p expression, indicating that miR-424-5p may act as an inhibitor of ferroptosis (Figures 1A, 1B). Furthermore, downregulation of miR-424-5p via its inhibitor remarkably enhanced erastin- and RSL3-induced cell death in both cell lines, whereas erastin- and RSL3-induced cell death could be rescued by treating these cells simultaneously with ferrostatin-1 (Fer-1), a potent inhibitor of ferroptosis (Supplementary Figure S2). Since lipid peroxidation and $\mathrm{Fe}^{2+}$ accumula- tion are two key events in triggering ferroptosis, we further tested whether miR-424-5p affects these events in ferroptosis. We found that overexpression of miR-424-5p significantly inhibited the accumulation of MDA, a key end-product of lipid peroxidation, but not $\mathrm{Fe}^{2+}$ accumulation induced by erasin and RSL3 in ovarian cancer cells (Figures 1C, 1D), whereas downregulation of miR-424-5p remarkably elevated MDA accumulation and did not affect the $\mathrm{Fe}^{2+}$ level in ovarian cancer cells (Supplementary Figure S3). These results suggest that miR-424-5p participates in erastin- and RSL3-induced ferroptosis in ovarian cancer cells by regulation of lipid peroxidation, but not $\mathrm{Fe}^{2+}$ accumulation.

ACSL4 is a direct target of miR-424-5p. To further unveil the mechanism underlying the effect of miR-424-5p on ovarian cancer ferroptosis, we performed the bioinformatic analysis by two algorithms (TargetScan and miRanda) to identify potential targets of miR-424-5p. Both of two algorithms predicted ACSL4, a key mediator of ferroptosis, as a potential target of miR-424-5p, and the 3 '-UTR of ACSL4 mRNA contains a highly conserved binding site from position 1973 to 1980 for miR-424-5p seed sequence (Figure 2A). To validate whether ACSL4 is the target of miR-424-5p, we examined the effect of miR-424-5p on the protein level of ACSL4 by western blot. The results showed that the level of ACSL4 protein both in HO8910 and SKOV3 cells was significantly decreased or increased by overexpression and inhibition of miR-424-5p, respectively (Figure 2B). To further test whether ACSL4 is a direct target gene of miR-424-5p, we constructed a luciferase reporter vector containing wild-type ACSL4 3'-UTR with the miR-424-5p binding site (WT) or containing the mutant 3'-UTR(MUT).

Table1. Clinical correlation between ACSL4 expression and clinicopathological characteristics in ovarian cancer patients.

\begin{tabular}{lccc}
\hline \multirow{2}{*}{ Clinical characteristics } & \multicolumn{2}{c}{ ACSL4 expression } & \multirow{2}{*}{ p-value } \\
\cline { 2 - 3 } & Low $(\mathbf{n}=\mathbf{1 9})$ & High $(\mathbf{n}=\mathbf{1 9})$ & \\
\hline Age (years) & 11 & 6 & \\
$\quad<55$ & 8 & 13 & 0.1028 \\
$\quad \geq 55$ & & & \\
Grade & 12 & 6 & \\
$\quad 1 / 2$ & 7 & 13 & 0.0615 \\
$\quad 3$ & & & \\
FIGO stage & 16 & 8 & \\
$\quad$ I-II & 3 & 11 & $0.0256^{*}$ \\
$\quad$ III-IV & & & \\
Tumor size (cm) & 5 & 9 & \\
$\quad<5$ & 14 & 10 & 0.2206 \\
$\quad \geq 5$ & & & \\
Peritoneal metastasis & 7 & 15 & \\
$\quad$ Yes & 12 & 4 & $0.0028^{*}$ \\
$\quad$ No & & & \\
Nodal metastasis & 6 & 12 & \\
$\quad$ Yes & 13 & 7 & $0.0039^{*}$ \\
$\quad$ No & ${ }^{*}$ p $<0.05$ statistically significant difference & &
\end{tabular}


A
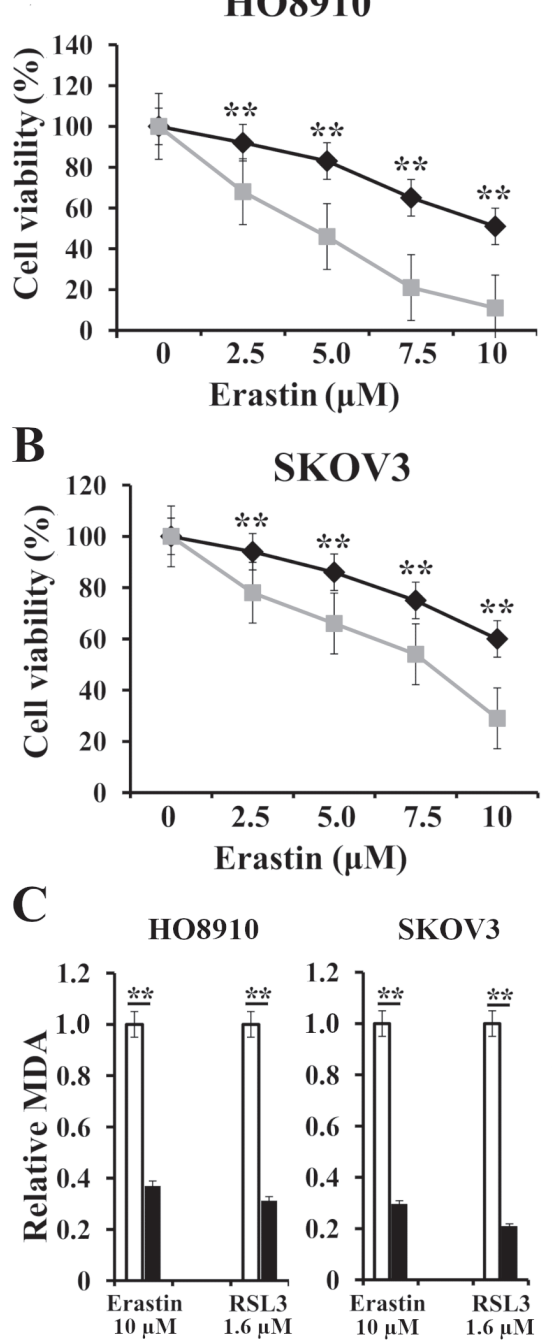

HO8910
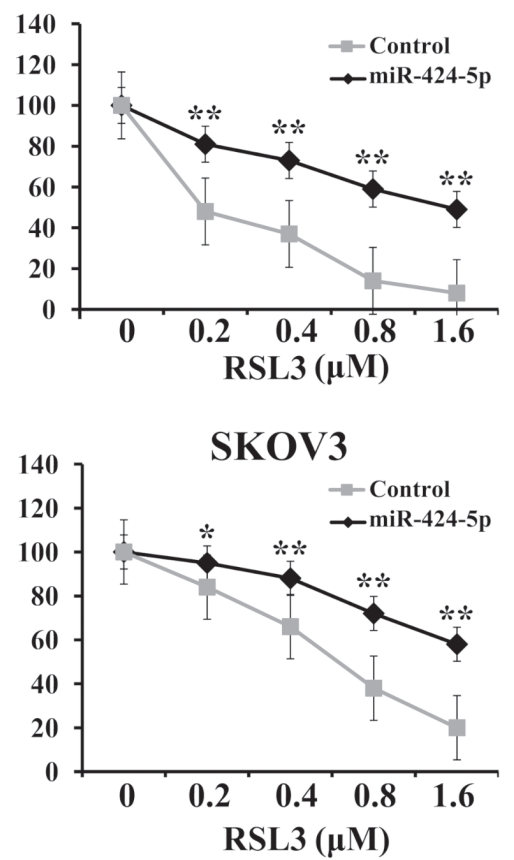

D

HO8910

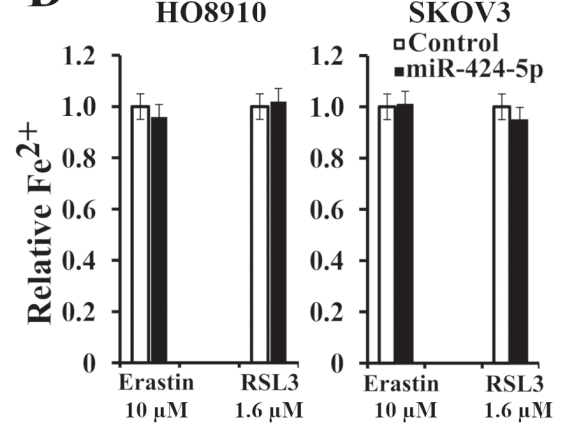

Figure 1. miR-424-5p negatively regulates ferroptosis in ovarian cancer. A, B) Upregulation of miR-424-5p inhibited erastin- and RSL3-induced cell death in HO8910 and SKOV3 cells. C) miR-424-5p overexpression significantly inhibited erastin- and RSL3-induced lipid peroxidation. D) miR-424$5 p$ overexpression did not affect erastin- and RSL3-induced $\mathrm{Fe}^{2+}$ accumulation. ${ }^{*} \mathrm{p}<0.05,{ }^{* *} \mathrm{p}<0.01$ compared with control group.

We found that miR-424-5p remarkably inhibited the luciferase activity of ACSL4-3'UTR-WT reporter, compared with control, but did not affect the mutant reporter both in HO8910 and SKOV3 cells (Figure 2C). To confirm whether the regulations described above in ovarian cancer cell lines are also clinically relevant, we further examined the relationship between expression of ACSL4 mRNA and miR-424-5p level in 38 ovarian cancer tissues by qRT-PCR. We found that ACSL4 mRNA was negatively associated with a miR-424-5p level in ovarian cancer tissues (Figure 2D). Given the ACSL4 level suppressed by miR-424-5p, we further test the effect of miR-424-5p on its enzymatic activity. We found that the upregulation of $\mathrm{miR}-424-5 \mathrm{p}$ resulted in a significant decrease in ACSL4 enzymatic activity in ovarian cancer cells, whereas inhibition of miR-424-5p led to a remarkable increase in
ACSL4 enzymatic activity (Figure 2E). Together, these results indicated that ACSL4 is a direct target of miR-424-5p in ovarian cancer.

ACSL4 is upregulated in ovarian cancer and sensitizes ovarian cancer cells to ferroptosis. The upregulation of ACSL4 was reported in numerous types of cancer and the inhibition of ACSL4 suppressed ferroptosis in cancer cells. However, to the best of our knowledge, the expression level of ACSL4 and its effect on ferroptosis in ovarian cancer has not been investigated. We first tested the expression of ACSL4 mRNA in 38 pairs of ovarian cancer tissues and their matched adjacent normal tissues by qRT-PCR. As shown in Figure 3A, ACSL4 mRNA was significantly increased in ovarian cancer tissues compared with the paired-adjacent normal tissues. Moreover, compared with adjacent normal 
A

\begin{tabular}{lr} 
miR-424-5p & AAGUUUUGUACUUAACGACGA \\
& |||||| \\
ACSL4 3'UTR & CACUUAAGGUUGGGUGCUGCU \\
\hline
\end{tabular}

CACUUAAGGUUGGGGAACCAG

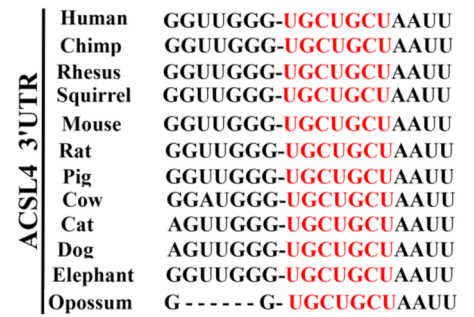

C

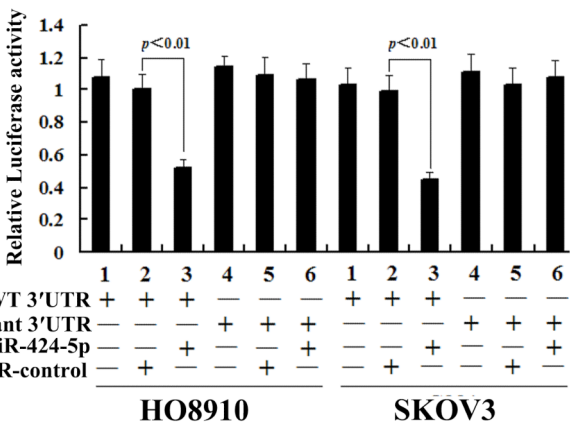

B
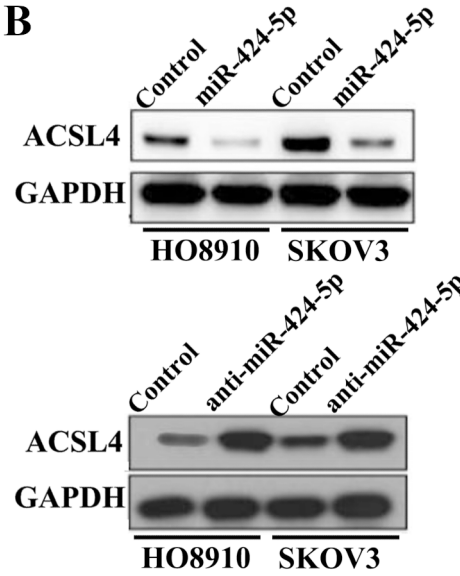

D

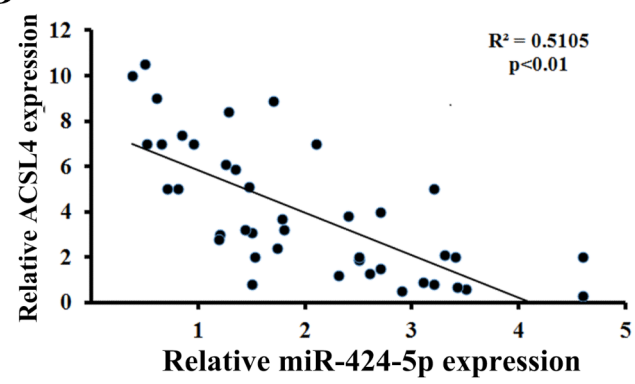

$\mathbf{E}$

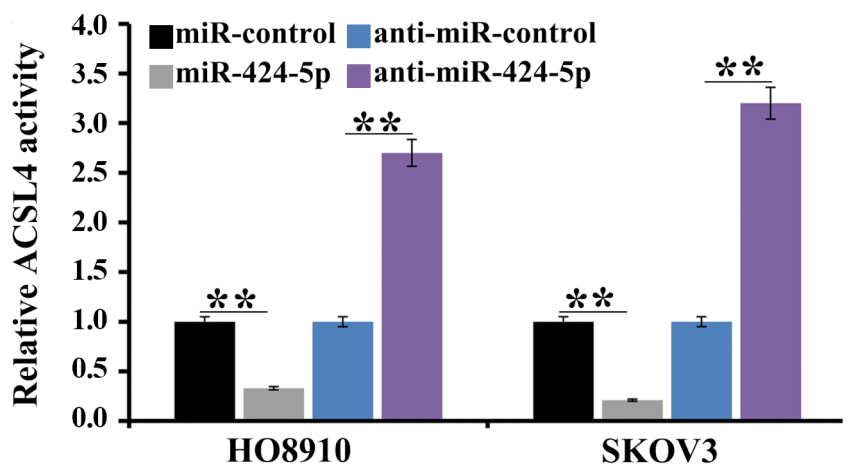

Figure 2. ACSL4 is a direct target of miR-424-5p. A) Sequence alignment of miR-424-5p and 3'-UTR of ACSL4. The seed sequence of miR-424-5p and binding sites in 3'-UTR are indicated in red. The 3'-UTR mutant containing mismatched nucleotides are shown at the bottom. The binding site of ACSL4 is highly conserved in several species. B) The protein levels of ACSL4 were affected by miR-424-5p in HO8910 and SKOV3 cells. C) miR-424-5p inhibited the expression of 3'-UTR-luciferase reporter of ACSL4 in HO8910 and SKOV3 cells, but did not affect that of the mutant construct. D) miR424-5p levels were negatively related to mRNA levels of ACSL4 in ovarian cancer tissues. E) miR-424-5p inhibited the enzymatic activity of ACSL4 in ovarian cancer cells.

tissues, upregulation of ACSL4 was also confirmed in the above mentioned ovarian cancer tissues by IHC assay (Supplementary Figure S4A).

Next, the correlation between ACSL4 expression and clinicopathological parameters was further investigated in 38 ovarian cancer patients, which were divided into two groups by the median level of ACSL4. The results revealed that ACSL4 expression was positively associated with the tumor stage and metastasis while no significant correlation was found in other parameters (Table1). Furthermore, patients with high expression of ACSL4 had significantly shorter overall survival times compared with those with low ACSL4 expression (Figure 3B). To further validate these results, we used the UALCAN online tool (http://ualcan. path.uab.edu/index.html) to analyze the ACSL4 protein level in ovarian cancer and non-tumor ovarian tissues. Consistent 

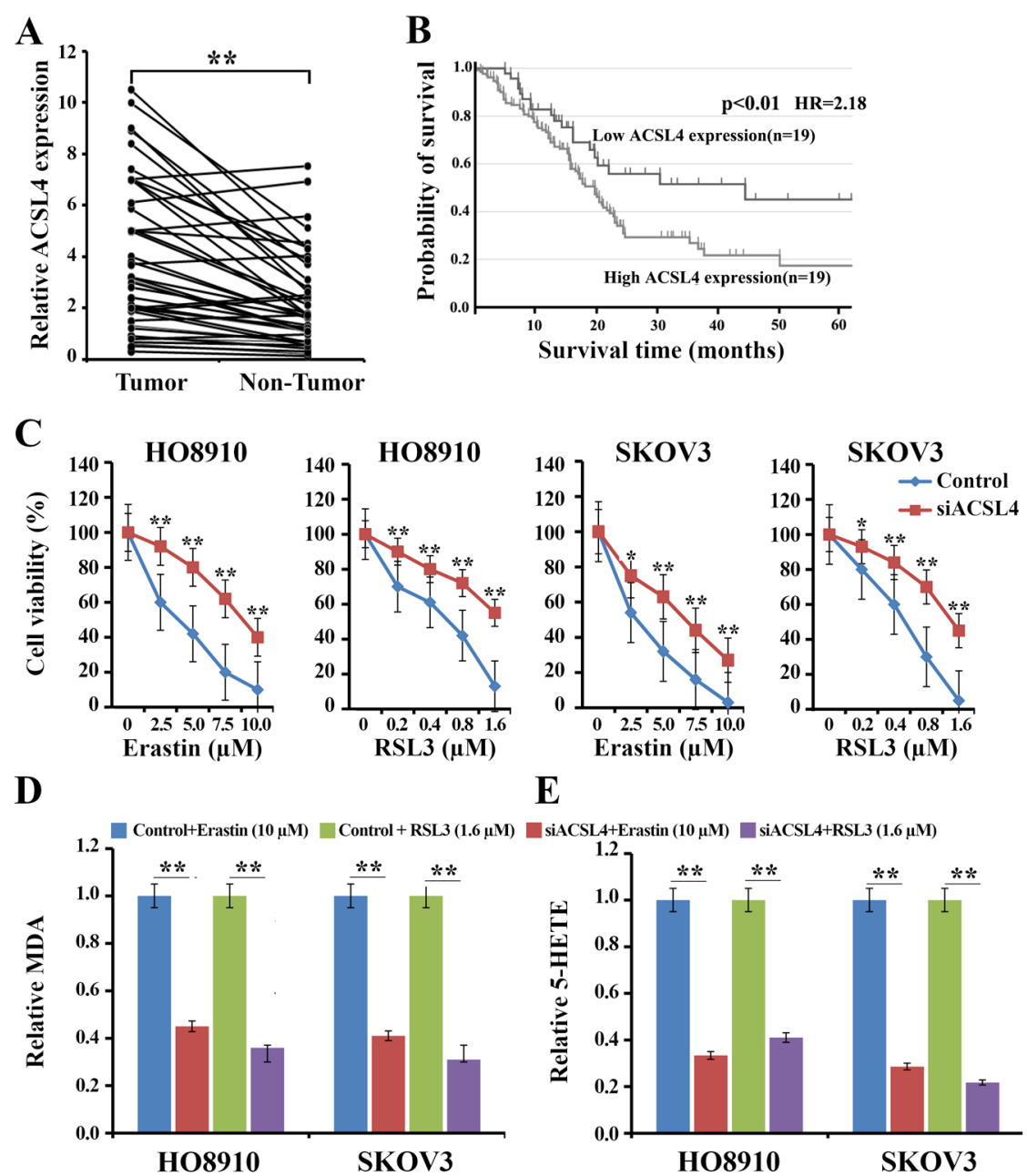

E

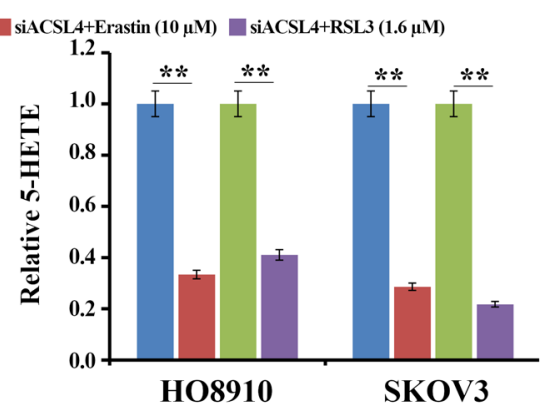

Figure 3. ACSL4 is upregulated in ovarian cancer and is associated with poor outcome and sensitized ovarian cancer cells to erastin- and RSL3-induced ferroptosis. A) The mean expression level of ACSL4 in ovarian cancer tissues was significantly higher than that in pair-matched adjacent non-cancerous normal tissues. B) Kaplan-Meier curves of the overall survival of $\mathbf{3 8}$ ovarian cancer patients were scored as low expression level and high expression level according to the ACSL4 expression. The ACSL4 upregulation was significantly correlated with overall shorter survival. C) Knockdown of ACSL4 inhibited erastin- and RSL3-induced ovarian cancer cell death. D) Knockdown of ACSL4 inhibited erastin- and RSL3-induced lipid peroxidation. E) Knockdown of ACSL4 inhibited erastin- and RSL3-induced 5-HETE production.

with our findings, the result showed that protein expression of ACSL4 in ovarian cancer was significantly higher than that in normal tissues (Supplementary Figure S4B). Additionally, we performed Kaplan-Meier analysis by the KaplanMeier Plotter online tool (http://kmplot.com/analysis/) and found that high ASCL4 expression is associated with shorter overall survival in ovarian cancer patients (Supplementary Figure S4C).

Finally, we further explore whether ACSL4 enhanced the sensitivity of ovarian cancer cells to erastin- and RSL3-induced ferroptosis. Downregulation of ACSL4 by its specific siRNAs significantly decreased the sensitivity to erastin- and RSL3-induced cell death in HO8910 and SKOV3 cells (Figure 3C). Moreover, we examined the level of lipid peroxidation and 5-HETE, an ACSL4-mediated product of arachidonic acid oxidation which contributes to ferroptosis, in ovarian cancer cells following the suppression of ACSL4. We found that the knockdown of ACSL4 significantly decreased erastin- and RSL3-induced MDA and 5-HETE production (Figures 3D, 3E). Collectively, these results suggested that ACSL4 is upregulated in ovarian cancer, which is associated with poor prognosis and enhances the sensitivity of ovarian cancer cells to erastinand RSL3-induced ferroptosis.

ACSL4 impairs miR-424-5p mediated ferroptosis inhibition in ovarian cancer cells. We further investigated whether miR-424-5p participated in ferroptosis via targeting ACSL4. To this end, HO8910 and SKOV3 cells were co-transfected with both miR-424-5p mimics and ACSL4 cDNA (Figure 4A). The results showed that miR-424-5p significantly 

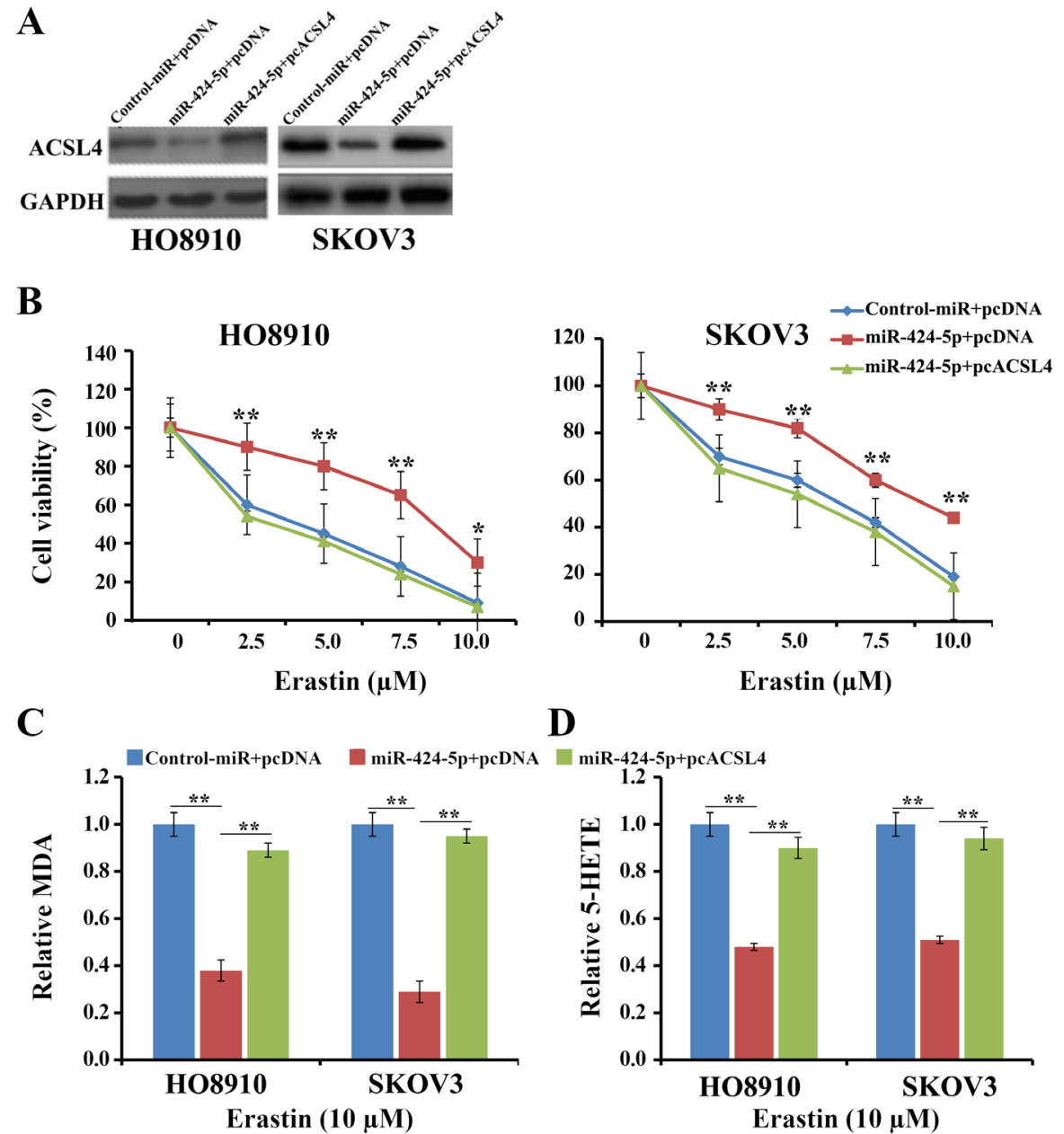

D

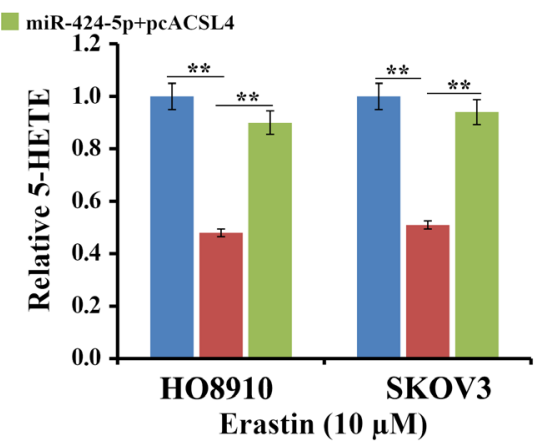

Figure 4. Overexpression of ACSL4 restored miR-424-5p mediated ferroptosis inhibition. A) Western blotting analysis of ACSL4 expression in HO8910 and SKOV3 cells transfected with both miR-424-5p and ACSL4 cDNA. B) Overexpression of ACSL4 reversed the inhibitory effect of miR-424-5p on erastin-induced cell death. C) Overexpression of ACSL4 reversed the inhibitory effect of miR-424-5p on erastin-induced lipid peroxidation. D) Overexpression of ACSL4 reversed the inhibitory effect of miR-424-5p on erastin-induced 5-HETE production.

inhibited erastin- and RSL3-induced cell death, MDA, and 5-HETE production, but this inhibitory effect was reversed by overexpression of ACSL4 (Figures 4C, 4D and Supplementary Figure S5). Taken together, these data indicated that miR-424-5p suppresses ferroptosis by regulating the expression of ACSL4 in ovarian cancer cells.

\section{Discussion}

miR-424-5p functions as an important tumor suppressor in multiple types of cancer including ovarian cancer [23-25]. Several previous studies have shown that downregulation of certain tumor suppressor miRNAs can sensitize cancer cells to induce ferroptosis. In this study, we expand these findings to
miR-424-5p which participates in erastin- and RSL3-induced ferroptosis in ovarian cancer. ACSL4, a positive mediator of ferroptosis, is a direct target of miR-424-5p. The upregulation of ACSL4 reverses the ferroptosis-suppressing effect of miR-424-5p, suggesting that miR-424-5p regulates ferroptosis in ovarian cancer by its inhibitory effects on ACSL4.

ACSL4, a member of the Acyl-CoA synthetase long-chain family, is an important enzyme responsible for catalyzing fatty acids with a backbone of 12-20 carbons to synthesize fatty acyl-CoAs. Accumulating evidence has demonstrated that ACSL4 is upregulated in a variety of cancers, which promotes an aggressive phenotype and resistance to conventional treatments through dysregulation of lipid metabolism [26-28]. Thus, targeting ACSL4 could be a key therapeutic approach for cancer treatment. Interestingly, ACSL4 has also 
been identified as a biomarker and contributor of ferroptosis by enriching cellular membranes with long polyunsaturated $\omega 6$ fatty acids which are the most susceptible lipids to peroxidation in the course of ferroptosis [21]. Furthermore, the ACSL4 expression was positively associated with sensitivity to ferroptosis induction [29]. These studies suggested that ACSL4 is required for maintaining the aggressive phenotypes of malignant cells in normal conditions, but it facilitates triggering ferroptosis following erastin and RSL3 treatment. Consistent with these previous studies, our studies found that ACSL4 was remarkably overexpressed in ovarian cancer tissues and positively correlated with the aggressive phenotypes of ovarian cancer. Most importantly, we found that ACSL4 knockdown could abrogate the sensitivity of ovarian cancer cells to erastin- and RSL3-induced ferroptosis. Therefore, our data for the first time provide evidence that induction of ferroptosis in ovarian cancer with ACSL4 overexpression may be regarded as a potential and novel therapeutic strategy for ovarian cancer treatment.

miR-424-5p plays a crucial role in carcinogenesis as a tumor suppressor in various types of cancer. Several studies have also shown that miR-424-5p was downregulated in ovarian cancer. As a target of miR-424-5p, ACSL4 was found upregulation in multiple types of cancer, such as colorectal cancer, breast cancer, and hepatocellular carcinoma so on [30-32]. Consistently, our results revealed that ACSL4 was increased in ovarian cancer and negatively correlated with the expression of miR-424-5p. All these pieces of evidence suggested that ACSL4 is a key target of miR-424-5p to suppress carcinogenesis. Accumulating data has demonstrated that miR-424-5p not only participated in cancer pathogenesis and progression but also is involved in drug resistance in many types of cancer. For example, the silencing of miR-424-5p induces radiotherapy resistance by targeting the lncRNA PVT1/ CARM1 axis in non-small-cell lung cancer [33]. Repression of miR-424 significantly inhibits chemosensitivity to cisplatin in gastric cancer by targeting SMURF1 [34]. Restoration of miR-424 sensitizes chronic myeloid leukemia cells to imatinib by targeting BCR-ABL [35]. Recently, some studies have indicated that downregulation of tumor suppression miRNAs could sensitize the cancer cells to ferroptosis induction, although it contributes to cancer progression and chemoresistance. In our study, we focus on the effect of miR-424-5p on the regulation of ferroptosis. We found that knockdown or overexpression of miR-424-5p could sensitize and suppress ovarian cancer cells to erastin- and RSL3-induced ferroptosis, respectively. Especially, downregulation of miR-424-5p increased ACSL4 expression, a positive mediator of ferroptosis, which facilitated ferroptotic cell death. Collectively, these observations revealed a novel regulatory mechanism by which the miR-424-5p/ACSL4 axis is involved in ferroptosis. These results also provided a potential therapeutic strategy in which ovarian cancer cells with downregulation of miR-424-5p may be particularly vulnerable to ferroptosis as opposed to other cell death mechanisms.

\section{References}

[1] TORRE LA, TRABERT B, DESANTIS CE, MILLER KD, RUNNOWICZ CD et al. Ovarian cancer statistics, 2018. CA Cancer J Clin 2018; 68: 284-296. https://doi.org/10.3322/ caac. 21456

[2] HERZOG TJ, MONK BJ. Bringing new medicines to women with epithelial ovarian cancer: what is the unmet medical need? Gynecol Oncol Res Pract 2017; 4: 13. https://doi. org/10.1186/s40661-017-0050-0

[3] PATCH AM, CHRISTIE EL, ETEMADMOGHADAM D, GARSED DW, GEORGE J et al. Whole-genome characterization of chemoresistant ovarian cancer. Nature 2015; 521: 489-494. https://doi.org/10.1038/nature14410

[4] CHAN JJ, TAY Y. Noncoding RNA: RNA Regulatory Networks in Cancer. Int J Mol Sci 2018; 19: E1310. https://doi. org/10.3390/ijms19051310

[5] BERINDAN-NEAGOE I, MONROIG PDC, PASCULLI B, CALIN GA. MicroRNAome genome: a treasure for cancer diagnosis and therapy. CA Cancer J Clin 2014; 64: 311-336. https://doi.org/10.3322/caac.21244

[6] HE LH, ZHU W, CHEN Q, YUAN YS, WANG YX et al. Ovarian cancer cell-secreted exosomal miR-205 promotes metastasis by inducing angiogenesis. Theranostics 2019; 9: 8206-8220. https://doi.org/10.7150/thno.37455

[7] BAGNOLI M, PIGNATA S, MEZZANZANICA D. A miRNA signature assessing ovarian cancer prognosis. Oncoscience 2016; 3: 308-310. https://doi.org/10.18632/oncoscience.329

[8] CHA SY, CHOI YH, HWANG S, JEONG JY, AN HJ. Clinical Impact of microRNAs Associated With Cancer Stem Cells as a Prognostic Factor in Ovarian Carcinoma. J Cancer 2017; 8: 3538-3547. https://doi.org/10.7150/jca.20348

[9] LIU JJ, GU ZP, TANG YJ, HAO JM, ZHANG CP et al. Tumour-suppressive microRNA-424-5p directly targets CCNE1 as potential prognostic markers in epithelial ovarian cancer. Cell Cycle 2018; 17: 309-318. https://doi.org/10.1080 /15384101.2017.1407894

[10] LI T, LI YM, GAN YQ, TIAN RT, WU QH et al. Methylationmediated repression of MiR-424/503 cluster promotes proliferation and migration of ovarian cancer cells through targeting the hub gene KIF23. Cell Cycle 2019; 18: 1601-1618. https://doi.org/10.1080/15384101.2019.1624112

[11] GOMAA A, PENG DF, CHEN Z, SOUTTO M, ABOUELEZZ $\mathrm{K}$ et al. Epigenetic regulation of AURKA by miR-4715-3p in upper gastrointestinal cancers. Sci Rep 2019; 9: 16970. https://doi.org/10.1038/s41598-019-53174-6

[12] ZHANG KX, WU LF, ZHANG P, LUO MY, DU J et al. miR9 regulates ferroptosis by targeting glutamic-oxaloacetic transaminase GOT1 in melanoma. Mol Carcinog 2018; 57: 1566-1576. https://doi.org/10.1002/mc.22878

[13] LUO MY, WU LF, ZHANG KX, WANG H, ZHANG T et al. miR-137 regulates ferroptosis by targeting glutamine transporter SLC1A5 in melanoma. Cell Death Differ 2018; 25: 1457-1472. https://doi.org/10.1038/s41418-017-0053-8

[14] TESFAY L, PAUL BT, KONSTORUM A, DENG ZY, COX AO et al. Stearoyl-CoA Desaturase 1 Protects Ovarian Cancer Cells from Ferroptotic Cell Death. Cancer Res 2019; 79: 5355-5366. https://doi.org/10.1158/0008-5472.CAN-19-0369 
[15] WORZFELD T, FINKERNAGEL F, REINARTZ S, KONZER A, ADHIKARY T et al. Proteotranscriptomics Reveal Signaling Networks in the Ovarian Cancer Microenvironment. Mol Cell Proteomics 2018; 17: 270-289. https://doi.org/10.1074/ mcp.RA117.000400

[16] KUCH EM, VELLARAMKALAYIL R, ZHANG I, LEHNEN D, BRUGGER B et al. Differentially localized acyl-CoA synthetase 4 isoenzymes mediate the metabolic channeling of fatty acids towards phosphatidylinositol. Biochim Biophys Acta 2014; 1841: 227-239. https://doi.org/10.1016/j.bbalip.2013.10.018

[17] DATTILO MA, BENZO Y, HERRERA L, PRADA JG, CASTILLO AF et al. Regulatory mechanisms leading to differential Acyl-CoA synthetase 4 expressionin breastcancercells. Sci Rep 2019; 9: 10324. https://doi.org/10.1038/s41598-019-46776-7

[18] SUN XJ, XU GL. Overexpression of Acyl-CoA Ligase 4 (ACSL4) in Patients with Hepatocellular Carcinoma and its Prognosis. Med Sci Monit 2017; 23: 4343-4350. https://doi. org/10.12659/msm.906639

[19] BELKAID A, OUELLETTE RJ, SURETTE ME. $17 \beta$-estradiolinduced ACSL4 protein expression promotes an invasive phenotype in estrogen receptor positive mammary carcinoma cells. Carcinogenesis 2017; 38: 402-410. https://doi. org/10.1093/carcin/bgx020

[20] DOLL S, PRONETH B, TYURINA YL, PANZILIUS E, KOBAYASHI $S$ et al. ACSL4 dictates ferroptosis sensitivity by shaping cellular lipid composition. Nat Chem Biol 2017; 13: 91-98. https://doi.org/10.1038/nchembio.2239

[21] YUAN H, LI XM, ZHANG XY, KANG R, TANG DL. Identification of ACSL4 as a biomarker and contributor of ferroptosis. Biochem Biophys Res Commun 2016; 478: 1338-1343. https://doi.org/10.1016/j.bbrc.2016.08.124

[22] ELLIS JM, LI LO, WU PC, KOVES TR, LLKAYEVA O et al. Adipose acyl-CoA synthetase-1 directs fatty acids toward beta-oxidation and is required for cold thermogenesis. Cell Metab 2010; 12: 53-64. https://doi.org/10.1016/j. cmet.2010.05.012

[23] WU JB, YANG B, ZHANG YP, FENG XD, HE B et al. miR424-5p represses the metastasis and invasion of intrahepatic cholangiocarcinoma by targeting ARK5. Int J Biol Sci 2019; 15: 1591-1599. https://doi.org/10.7150/ijbs.34113

[24] DU HM, XU Q, XIAO S, WU ZR, GONG JP et al. MicroRNA424-5p acts as a potential biomarker and inhibits proliferation and invasion in hepatocellular carcinoma by targeting TRIM29. Life Sci 2019; 224: 1-11. https://doi.org/10.1016/j. lfs.2019.03.028

[25] ZHOU Y, AN Q, GUO RX, QIAO YH, LI LX et al. miR424-5p functions as an anti-oncogene in cervical cancer cell growth by targeting KDM5B via the Notch signaling pathway. Life Sci 2017; 171: 9-15. https://doi.org/10.1016/j.lfs.2017.01.006
[26] SANCHEZ-MARTINEZ R, CRUZ-GIL S, GOMEZ DE CEDRON M, ALVAREZ-FERNANDEZ M, VARGAS T et al. A link between lipid metabolism and epithelial-mesenchymal transition provides a target for colon cancer therapy. Oncotarget 2015; 6: 38719-38736. https://doi.org/10.18632/oncotarget.5340

[27] ORLANDO UD, CASTILLO AF, MEDRANO MAR, SOLANO AR, MALOBERTI PM et al. Acyl-CoA synthetase-4 is implicated in drug resistance in breast cancer cell lines involving the regulation of energy-dependent transporter expression. Biochem Pharmacol 2019; 159: 52-63. https://doi. org/10.1016/j.bcp.2018.11.005

[28] WU XY, DENG FM, LI YR, DANIELS G, DU XX et al. ACSL4 promotes prostate cancer growth, invasion and hormonal resistance. Oncotarget 2015; 6: 44849-44863. https:// doi.org/10.18632/oncotarget.6438

[29] PARK SJ, OH JJ, KIM MH, JIN EJ. Bromelain effectively suppresses kras-mutant colorectal cancer by stimulating ferroptosis. Anim Cells Syst 2018; 22: 334-340. https://doi.org/10.1 080/19768354.2018.1512521

[30] CHEN WC, WANG CY, HUNG YH, WENG TY, YEN MC et al. Systematic analysis of gene expression alterations and clinical outcomes for long-chain acyl-coenzyme a synthetase family in cancer. PLoS ONE 2016; 11: e0155660. https://doi. org/10.1371/journal.pone. 0155660

[31] WU XY, LI YR, WANG JH, WEN X, MARCUS MT et al. Long chain fatty acyl-CoA synthetase 4 is a biomarker for and mediator of hormone resistance in human breast cancer. PLoS ONE 2013; 8: e77060. https://doi.org/10.1371/journal. pone. 0077060

[32] LIANG YC, WU CH, CHU JS, WANG CK, HUNG LF et al. Involvement of fatty acid-CoA ligase 4 in hepatocellular carcinoma growth: Roles of cyclic amp and p38 mitogenactivated protein kinase. World J Gastroenterol 2005; 11: 2557-2563. https://doi.org/10.3748/wjg.v11.i17.2557

[33] WANG D, HU Y. Long Non-coding RNA PVT1 Competitively Binds MicroRNA-424-5p to Regulate CARM1 in Radiosensitivity of Non-Small-Cell Lung Cancer. Mol Ther Nucleic Acids 2019; 16: 130-140. https://doi.org/10.1016/j. omtn.2018.12.006

[34] LU L, WU ML, LU YH, ZHAO ZC, LIU T et al. MicroRNA-424 regulates cisplatin resistance of gastric cancer by targeting SMURF1 based on GEO database and primary validation in human gastric cancer tissues. Onco Targets Ther 2019; 12: 7623-7636. https://doi.org/10.2147/OTT.S208275

[35] HERSHKOVITZ-ROKAH O, MODAI S, PASMANIKCHOR M, TOREN A, SHOMRON N et al. Restoration of miR-424 suppresses BCR-ABL activity and sensitizes CML cells to imatinib treatment. Cancer Lett 2015; 360: 245-256. https://doi.org/10.1016/j.canlet.2015.02.031 